\title{
Oxford MEST classification in Iranian patients with IgA nephropathy, with regards to extracapillary proliferation; a single center experience
}

\author{
Mohsen Akhavansepahi ${ }^{\circledR}$, Maryam Rafieyan², Hamid Nasri ${ }^{2 *(\mathbb{D}}$ \\ 'Department of Pediatric Nephrology, School of Medicine, Qom University of Medical Sciences, Qom, Iran \\ ${ }^{2}$ Department of Internal Medicine, Isfahan University of Medical Science, Isfahan, Iran
}

\section{Correspondence to:}

Prof. Hamid Nasri, Email:

hamidnasri@yahoo.com,

hamidnasri@med.mui.ac.ir

Received: 17 September 2020 Accepted: 3 Nov 2020 ePublished: 11 Nov. 2020

Keywords: IgA nephropathy, Endocapillary hypercellularity, Extracapillary proliferation Tubular atrophy/interstitial fibrosis, Crescent

\begin{abstract}
Introduction: IgA nephropathy is the most common glomerulonephritis in the world. Objectives: In this study we aimed to find the relationship between morphological lesions of Oxford classification for IgA nephropathy with clinical findings and some laboratory data. Patients and Methods: All kidney biopsy conducted from 2009 to 2019 conducted in medical centers and were sent to a reference laboratory in Isfahan. All kidney biopsies included two samples for immunofluorescence and light microscopy. After definitive diagnosis of $\operatorname{IgAN}$ (IgA nephropathy), the slides were examined to classify the disease based on the Oxford-MEST ( $M$; mesangial hypercellularity, E; endocapillary hypercellularity, S; segmental glomerulosclerosis, and $\mathrm{T}$; tubular atrophy/interstitial fibrosis and also C; crescent) classification.

Results: Our study on 238 biopsy proven IgA nephropathy patients showed that 78 patients $(32.8 \%)$ were male. Mean \pm SD of age individuals was $38.00 \pm 13.68$ years. The mean \pm SD of serum creatinine level was $1.42 \pm 0.79 \mathrm{mg} / \mathrm{dL}$ and the mean $\pm \mathrm{SD}$ of proteinuria was $1780.94 \pm 1168.75 \mathrm{mg} / \mathrm{d}$. Our study showed no significant association between $\mathrm{M}$, E and S variables with patients' age. However, a statistically significant relationship between $\mathrm{T}$ variable and patients' age $(P=0.028)$ was detected. Furthermore, morphologic variables of $\mathrm{M}, \mathrm{E}$ and $\mathrm{S}$ were not significantly associated with proteinuria while $\mathrm{T}$ variable was positively associated with the quantity of proteinuria $(P=0.021)$.

Conclusion: The association of tubular atrophy/interstitial fibrosis (T variable) with quantity of proteinuria showed significance of interstitial lesion on the prognosis IgA nephropathy.
\end{abstract}

Introduction

IgA nephropathy is the most common glomerulonephritis in the world (1). This disease is the deposition of $\operatorname{Ig} \mathrm{A}$ antibodies in the mesangial space of the glomeruli (2). The disease is mild in many patients, however a group of patients eventually develop end-stage renal disease (ESRD) and in $50 \%$ of cases (1-4). Its prevalence varies in different parts of the world from less than $10 \%$ in the United States to $30-40 \%$ in Asia (2-5). Nevertheless, the diagnosis percentage of this disease depends on the number of biopsies taken in each country. It is more common in whites and Asians and is twice as common in men as women. Most patients are in the age range of 16 to 35 years (2-6).

The range of symptoms of this disease varies from asymptomatic hematuria to rapidly progressive glomerulonephritis $(1,3,6)$. Other symptoms of gross hematuria include renal failure and hypertension [1-

\begin{abstract}
Key point
In a cross- sectional study on 238 biopsy proven IgA nephropathy patients, we found an association between tubular atrophy/interstitial fibrosis ( $T$ variable) and quantity of proteinuria, which imply the significance of interstitial lesion on the prognosis IgA nephropathy.
\end{abstract}

3]. The risk of chronic renal failure is not exactly predictable, however males who have proteinuria ( $\geq 2 \mathrm{~g}$ in 24 hours), hypertension (systolic blood pressure $\geq 130 \mathrm{~mm} \mathrm{Hg}$ and diastolic blood pressure $\geq 90 \mathrm{~mm} \mathrm{Hg}$ ), and early renal failure are some factors worsening the prognosis (3-6). Pathological diagnosis of $\operatorname{IgA}$ nephropathy requires observation of IgA deposition in the mesangial space, which is detected by immunofluorescence microscopy $(1,2)$.

Objectives

In this study, we aimed to find the relationship 
between morphological lesions of Oxford classification with clinical findings and laboratory data to find people with higher risk in the early stages.

\section{Patients and Methods}

\section{Study design}

Definitive diagnosis of $\operatorname{IgA}$ nephropathy requires mesangial IgA deposition (above +2 score intensity) in the mesangial or mesangiocapillary region alongside with very few or absence of C1q deposits $(1,2,5)$.

All kidney biopsies were conducted from 20092019 in medical centers of Isfahan and Chahar-Mohal \& Bakhtyari provinces and were sent to a reference laboratory. This study is an extension of our previous study conducted in 2012 on IgA nephropathy. Our previous study was conducted on 102 patients (7). None of the patients were treated before the biopsy taking. All 246 patients, enrolled in this study, showed predominant IgA deposition, on the biopsy under immunofluorescence microscopy in addition to morphologic findings of this disease in light microscopy. In our study, IgA deposits scored from 0 to 3 , depending upon the intensity of brightness of the antibody under the immunofluorescence microscopy. According to the questionnaire filled out at the time of referral, of 246 samples with definitive diagnosis of IgA nephropathy, 8 patients were not evaluated from the beginning due to concomitant diabetes. Additionally, biopsies containing less than 8 glomeruli were not included in the study from the beginning. Finally, the study was performed on 238 patients. All kidney biopsies included two samples for immunofluorescence and light microscopy. One sample was placed in $10 \%$ formalin for staining of hematoxylin and eosin, periodic acid Schiff, Masson's trichrome and Jones methenamine silver. The second sample was placed in normal saline for rapid examination by immunofluorescence microscopy to assess the depositions of $\mathrm{C} 1 \mathrm{q}, \mathrm{C} 3, \operatorname{IgG}, \operatorname{IgA}$ and $\operatorname{IgM}$ antibodies according to a score of 0 to 3 , depending upon the intensity of brightness of the antibody. Immunofluorescence study was conducted without knowledge on laboratory or clinical data of the patients. Immunofluorescence study was also conducted before the light microscopic examination of specimens. The slides were examined by a nephropathologist. After definitive diagnosis of $\operatorname{IgAN}$ (IgA nephropathy), the slides were examined to classify the disease based on the modified Oxford-MEST classification.

Revised Oxford classification for IgA nephropathy called MEST-C, is the modified version of which was used in this study (1). MEST includes the following morphologic variables:

M: mesangial hypercellularity

E: endocapillary hypercellularity

S: segmental glomerulosclerosis

T; tubular atrophy/interstitial fibrosis

C: extra-capillary proliferation (crescent).

In addition, age, gender, serum creatinine $(\mathrm{Cr})$ and proteinuria (in 24-hour urine) were the data recorded for each patient at biopsy taking time.

\section{Ethical issues}

The research followed the tenets of the Declaration of Helsinki. The institutional ethical committee at Isfahan University of Medical Science approved all study protocols (\#IR.MUI.MED.REC.1397.163). Accordingly, written informed consent was taken from all participants before any intervention. This study is a part of M.D thesis of Maryam Rafieyan at this university (Thesis\# 397476).

\section{Data analysis}

Data were entered into SPSS software version 20. For qualitative variables, the results were entered in frequency tables and frequency percentage was calculated. Chisquare test was used to determine qualitative variables and ANOVA analysis was used to interpret quantitative variables (age, serum creatinine and proteinuria). $P<0.05$ was considered as the significant level.

\section{Results}

This is an observational study conducted on 238 biopsy proven IgA nephropathy patients. Seventy-eight patients (32.8\%) were male, since 11 patients $(4.6 \%)$ were under 20 years old. Around 141 patients (59.2\%) were between 20 to 40 years old and the remaining 86 patients $(36.1 \%)$ were over 40 years old. The maximum and minimum age of patients was 85 years and 15 years, respectively. Mean \pm SD of age individuals was $38.00 \pm 13.68$ years (median value of age was 35 years). The mean \pm SD of serum creatinine level was $1.42 \pm 0.79 \mathrm{mg} / \mathrm{dL}$. Moreover, the mean $\pm \mathrm{SD}$ of proteinuria was $1780.94 \pm 1168.75 \mathrm{mg} / \mathrm{d}$ (Table 1).

In this study, $70.2 \%$ of patients had mesangial hypercellularity (M1), 36.1\% had endocapillary lesion (E1) and $64.7 \%$ had segmental glomerulosclerosis (S1). Accordingly, $36.1 \%$ and $20.2 \%$ had $\mathrm{T} 1$ and $\mathrm{T} 2$ (tubular atrophy/interstitial fibrosis) respectively. Furthermore, at the time of biopsy, 69 patients (29\%) had crescents (Table 2).

\section{Relationship between MEST-C variables and serum creatinine}

In our study, E, $S$ and variables had a significant relationship with serum creatinine $(P<0.05)$, since the relationship of $M$ variable with serum creatinine was not significant $(P=0.078)$

\section{Relationship between MEST-C variables with age and proteinuria}

There was no association between M0, M1, E and S with age. However, a statistically significant relationship between $\mathrm{T}$ variable and age $(P=0.028)$ was detected (ANOVA analysis). There was no relationship between age and crescent's number. Furthermore, morphologic variables of $\mathrm{M}, \mathrm{E}$ and $\mathrm{S}$ were not significantly associated 
Table 1. Demographic information

\begin{tabular}{lll}
\hline & Mean \pm SD & Median $[$ min, max] \\
\hline Age (years) & $38.00 \pm 13.68$ & $35[15,85]$ \\
\hline Serum creatinine $(\mathrm{mg} / \mathrm{dL})$ & $1.42 \pm 0.79$ & $1.2[0.5,6.5]$ \\
Proteinuria $(\mathrm{mg} / \mathrm{d})$ & $1780.94 \pm 1168.75$ & $1800[200,9000]$ \\
Number of crescent & $0.47 \pm 0.90$ & $0[0.6]$ \\
Interstitial fibrosis $(\%)$ & $13.97 \pm 16.18$ & $10[0.75]$ \\
\hline
\end{tabular}

Table 2. Frequency of MEST variables

\begin{tabular}{llc}
\hline MEST & & No. $(\%)$ \\
\hline \multirow{2}{*}{ M } & M1 & $167(70.2)$ \\
E & M0 & $71(29.8)$ \\
& E1 & $86(36.1)$ \\
S & E0 & $152(63.9)$ \\
& S1 & $154(64.7)$ \\
\multirow{2}{*}{ T } & S0 & $84(35.3)$ \\
& T0 & $104(43.7)$ \\
\hline
\end{tabular}

E (endocapillary hypercellularity), S (segmental sclerosis), tubular atrophy/ interstitial fibrosis $(T)$, mesangial hypercellularity $(M)$.

with proteinuria; however, $\mathrm{T}$ variable was positively associated with the quantity of proteinuria $(P=0.021)$.

\section{Discussion}

The average proteinuria in our present study was $1.5 \mathrm{~g} /$ day, which was lower than the quantity reported by Monteiro et al $(1.8 \mathrm{~g} / \mathrm{d})(7)$. In their study, $63.11 \%$ of patients had significant proteinuria (more than one gram per 24 hours), which was similar to our results (8). In our study, unlike the study by dos Reis Monteiro et al, no positive relationship was found between the S variables of patients' age and proteinuria (8). In the study of Riispere et al, the mean age of patients was 33.7 years and in the recent study was 38 years. There was no significant relationship between $\mathrm{E}$ and proteinuria in the study by Riispere et al (9). In our study, $70.2 \%$ had mesangial hypercellularity, $36.1 \%$ endocapillary lesion, $64.7 \%$ segmental glomerulosclerosis and $56.3 \%$ tubular atrophy/interstitial fibrosis (T1/2) while in the study by Bagchi et al, 77.7\%, 9.7\%, 43.7\% and 39.8\% of patients showed M1, E1, S1 and T1/T2, respectively (10). In our study, $29 \%$ of patients had crescent while in the study by Markowitz et al crescents were found in 36\% of biopsy specimens (11). The study of Mizerka-Wasiak et al on children with IgAN, showed E and S had statistically significant relationship with proteinuria, however in our study no association of $\mathrm{M}, \mathrm{E}$ and $\mathrm{S}$ variables with proteinuria was observed while among the variables only $\mathrm{T}$ had a positive significant relationship with quantity of proteinuria $(P=0.021)(12)$. As mentioned, the frequency of $\mathrm{M} 1, \mathrm{E} 1, \mathrm{~S} 1$ and $\mathrm{T} 1 / 2$ in our study were $\mathrm{M} 1 ; 70.2 \%$,
$\mathrm{E} 1 ; 36.1 \%, \mathrm{~S} 1 ; 64.7 \%$ and $\mathrm{T} 1 / 2$ was $56.3 \%$, while in the study of $\mathrm{Gu}$ et al, the percentages of M1, E1, S1 and T1/ 2 were $97.6 \%, 14.6,50$ and $68.6 \%$, respectively (13). This study also showed, the mean age of patients was 32 years while $53 \%$ of patients were female, while in our study, the mean age of patients was 38 years and $67.2 \%$ of patients were female. The mean proteinuria in a previous study was $1000 \mathrm{mg} / \mathrm{d}$ since in our study was, the median value was $1800 \mathrm{mg} / \mathrm{d}$ (14).

Conclusion

Our study on 238 biopsy-proven IgA nephropathy patients, showed no association between $\mathrm{M}, \mathrm{E}$ and $\mathrm{S}$ variables with patients' age. However, a statistically significant relationship between $\mathrm{T}$ variable and patients' age $(\mathrm{P}=$ $0.028)$ was detected. There was no relationship between age and crescent's number. Furthermore, morphologic variables of $\mathrm{M}, \mathrm{E}$ and $\mathrm{S}$ were not significantly associated with proteinuria while $\mathrm{T}$ variable was positively associated with the quantity of proteinuria.

Limitation of the study

This investigation is a single-center study. Larger specimens are required for appropriate generalization of data.

Authors' contribution

HN and MRK designed the research. MRK gathered the data and prepared the final draft in Persian. HN edited the final draft. MAS, translated the paper to English and conducted an extensive edition. All authors read and signed the final manuscript.

Ethical considerations

Ethical issues (including plagiarism, data fabrication, double publication) have been completely observed by the authors.

Conflicts of interest

The authors declare that they have no competing interests.

Funding/Support

None.

\section{References}

1. Trimarchi H, Barratt J, Cattran DC, Cook HT, Coppo R, Haas M, et al. IgAN Classification Working Group of the International IgA Nephropathy Network and the Renal Pathology Society; Conference Participants. Oxford Classification of IgA nephropathy 2016: an update from the IgA Nephropathy Classification Working Group. Kidney Int. 2017;91:1014-21. doi: 10.1016/j.kint.2017.02.003.

2. Roberts IS. Oxford classification of immunoglobulin A nephropathy: an update. Curr Opin Nephrol Hypertens. 2013;22:281-6. doi: 10.1097/MNH.0b013e32835fe65c.

3. Coppo R, Troyanov S, Bellur S, Cattran D, Cook HT, Feehally J, et al. VALIGA study of the ERA-EDTA Immunonephrology Working Group. Validation of the Oxford classification of IgA nephropathy in cohorts with different presentations and treatments. Kidney Int. 2014;86:828-36. doi: 10.1038/ ki.2014.63.

4. Barbour SJ, Espino-Hernandez G, Reich HN, Coppo R, Roberts IS, Feehally J, et al. Oxford Derivation, North American Validation and VALIGA Consortia; Oxford Derivation North American Validation and VALIGA Consortia. The MEST score 
provides earlier risk prediction in IgA nephropathy. Kidney Int. 2016;89:167-75. doi: 10.1038/ki.2015.322.

5. Soares MF, Roberts IS. IgA nephropathy: an update. Curr Opin Nephrol Hypertens. 2017;26:165-171. doi: 10.1097/ MNH.0000000000000312.

6. Alamartine E, Sauron C, Laurent B, Sury A, Seffert A, Mariat C. The use of the Oxford classification of IgA nephropathy to predict renal survival. Clin J Am Soc Nephrol. 2011;6:2384-8. doi: 10.2215/CJN.01170211.

7. Nasri H, Mortazavi M, Ghorbani A, Shahbazian H, Kheiri S, Baradaran A, et al. Oxford-MEST classification in IgA nephropathy patients: A report from Iran. J Nephropathology. 2012;1(1):31-42.

8. Monteiro MLGDR, Vieira MR, Pereira LHM, Araújo LS, Silva CA, Araújo LB, et al. Is it possible to predict parameters of the Oxford classification of primary IgA Nephropathy from clinical laboratory data? Focus on the role of segmental glomerulosclerosis subtypes. Pathol Res Pract. 2019;215:152533. doi: 10.1016/j.prp.2019.152533.

9. Riispere Ž, Laurinavičius A, Kuudeberg A, Seppet E, Sepp $\mathrm{K}$, Ilmoja $\mathrm{M}$, et al. IgA nephropathy clinicopathologic study following the Oxford classification: Progression peculiarities and gender-related differences. Medicina (Kaunas). 2016;52(6):340-8. doi: 10.1016/j.medici.2016.11.003.

10. Bagchi S, Singh G, Yadav R, Kalaivani M, Mahajan S, Bhowmik D, et al. Clinical and histopathologic profile of patients with primary IgA nephropathy seen in a tertiary hospital in India. Ren Fail. 2016;38:431-6. doi: 10.3109/0886022X.2016.1138817.

11. Markowitz G. Glomerular disease: updated oxford classification of IgA nephropathy: a new MEST-C score. Nat Rev Nephrol. 2017;13:385-6. doi: 10.1038/nrneph.2017.67.

12. Mizerska-Wasiak M, Małdyk J, Rybi-Szumińska A, Wasilewska A, Miklaszewska M, Pietrzyk J, et al. Relationship between serum IgA/C3 ratio and severity of histological lesions using the Oxford classification in children with IgA nephropathy. Pediatr Nephrol. 2015;30:1113-20. doi: 10.1007/s00467014-3024-z.

13. Gu Y, Wang Y, Ji C, Fan P, He Z, Wang T, et al. Syndrome differentiation of IgA nephropathy based on clinicopathological parameters: a decision tree model. Evid Based Complement Alternat Med. 2017;2017:2697560. doi: $10.1155 / 2017 / 2697560$ 